\title{
Der Einfluss der europäischen und nationalen Haushaltsregeln auf das deutsche Gesundheitswesen
}

\section{JOHANNES CLEMENS}

Johannes Clemens ist bei der Deutsche Bundesbank in Frankfurt am Main im Zentralbereich Volkswirtschaft tätig

\author{
Haushaltsregeln sollen die fiskalische Nachhaltigkeit \\ sicherstellen, und der kurzfristig leichteste Weg in die \\ Verschuldung soll verbaut werden. Der Rücklagen- \\ verzehr in der gesetzlichen Krankenversicherung wird \\ in den kommenden Jahren das gesamtstaatliche Defizit \\ erhöhen, das auch für den europäischen Fiskalpakt \\ maßgeblich ist. Wenngleich eine Sanktionierung \\ letztlich wenig wahrscheinlich erscheint, droht doch \\ ein Reputationsverlust. Die Bundeszuschüsse an die \\ Sozialversicherung dürften daher auch in Zukunft \\ zur Disposition gestellt werden.
}

\section{Nationale und europäische Haushaltsregeln}

Regeln für die öffentlichen Haushalte, zu denen auch die Sozialversicherungen zählen, sollen deren Tragfähigkeit sicherstellen - nicht zuletzt um Konflikte mit der Geldpolitik zu vermeiden. Zweifel an der Tragfähigkeit sind laut Stabilitäts- und Wachstumspakt grundsätzlich dann angebracht, wenn die staatlichen Defizite in Relation zum Bruttoinlandsprodukt (BIP) $3 \%$ überschreiten oder die Schuldenquote über $60 \%$ steigt. Fast alle Mitgliedstaaten der EU (Ausnahmen: Vereinigtes Königreich und Tschechische Republik) haben sich darüber hinaus mit dem Fiskalpakt verpflichtet, Teile des europäischen Stabilitäts- und Wachstumspaktes auch im nationalen Recht zu verankern. Die Regel legt insbesondere fest, dass mittelfristig ein strukturelles, das heißt um konjunkturelle und temporäre Einflüsse bereinigtes Defizit von im Regelfall 0,5\% des BIP nicht überschritten wird. Außerdem muss vorgesehen sein, dass im Falle eines Anstiegs der Schuldenquote über $60 \%$ hinaus, eine
Rückführung um mindestens $1 / 20$ pro Jahr eingeleitet wird.

In Deutschland begrenzt darüber hinaus die neue grundgesetzliche Schuldenregel die strukturelle Nettokreditaufnahme des Bundes ab 2016 auf höchstens 0,35 \%, und die Länder müssen ab 2020 strukturell ausgeglichene Haushalte aufweisen. Das Zusammenspiel der europäischen und nationalen Haushaltsregeln bedeutet, dass nach Ausschöpfen des maximalen nationalen Neuverschuldungspotenzials durch den Bund (0,35\% ab 2016) und die Länder ( $0 \%$ ab 2020) rein rechnerisch ein Spielraum von maximal $0,15 \%$ bis zum Erreichen der Haushaltsgrenze des Fiskalpaktes verbleibt. Derzeit entspricht dies einem Betrag von rund $4 \operatorname{Mrd} €$.

Für die Haushalte der Gemeinden und der Sozialversicherungen sehen weder die europäischen noch die deutschen Haushaltsregeln spezifische Begrenzungen vor. Allerdings sind beide Ebenen in die gesamtstaatlichen Vorgaben einbezogen und in ihren Verschuldungsmöglichkeiten durch anderweitige nationale Vorgaben deutlich eingeschränkt. 
Den Gemeinden sind haushaltsmäßige Kreditaufnahmen grundsätzlich nur zur Finanzierung von Investitionen im Rahmen ihrer wirtschaftlichen Leistungsfähigkeit gestattet. Dementsprechend müssen sie die Abschreibungen bzw. die zur Tilgung der Altschulden notwendigen Mittel erwirtschaften. Somit kann sich ihre Nettovermögensposition in diesem Rahmen grundsätzlich nicht verschlechtern. Vielfach konnten die notwendigen Mittel in der Vergangenheit allerdings nicht (vollständig) erwirtschaftet werden. In einzelnen Jahren traten offenbar sogar Defizite im Bereich der laufenden Ausgaben auf, zu deren Finanzierung insbesondere in einigen westdeutschen Ländern auch verstärkt auf Kassenkredite zurück gegriffen wurde.

Den Sozialversicherungen ist es grundsätzlich nicht erlaubt, sich über Kredite zu finanzieren. Für die gesetzlichen Krankenkassen schreibt $\$ 220$ Abs. 1 SGB V explizit vor, dass Darlehensaufnahmen nicht zulässig sind. ${ }^{1}$ Defizite sind in der gesetzlichen Krankenversicherung gleichwohl dann möglich, wenn Rücklagen abgebaut werden, die durch zuvor erzielte Überschüsse gebildet worden sind. In der Vergangenheit ergaben sich wiederholt Defizite bei den Sozialversicherungen, die bis an 0,4\% des BIP heranreichten (z.B. im Jahr 2002). Im Krisenjahr 2009 betrug deren Defizit sogar über 0,6\% des BIP. Bereinigt man (näherungswei$\left.\mathrm{se}^{2}\right)$ um die konjunkturellen Einflüsse auf der Einnahmenseite (das heißt um zyklische Entgelt- und Beschäftigungsschwankungen), so zeigt sich seit 2003 für die Sozialversicherungen kein Überschreiten des maximalen rechnerischen Spielraums von $0,15 \%$ des BIP mehr. Dies ist freilich keine Garantie dafür, dass es in Zukunft auch so bleibt.

Die Finanzen der gesetzlichen Krankenversicherung können über den konjunkturelles Zyklus hinweg „atmen“, soweit und solange Rücklagen vorhanden sind. Persistente strukturelle Defizite sind daher zwar grundsätzlich nicht zu erwarten, da eine Schuldenfinanzierung gesetzlich ausgeschlossen ist und dem Abbau finanzieller Reserven zunächst wieder ein Aufbau folgen muss. Gleichwohl ist es durchaus möglich, dass Krankenkassen und Gesundheitsfonds in einzelnen Jahren das strukturelle gesamtstaatliche Defizit größer ausfallen lassen - etwa wenn die derzeit hohen Rücklagen demnächst abgeschmolzen werden. Ende 2012 belie- fen sich die Finanzreserven in der gesetzlichen Krankenversicherung auf insgesamt fast $281 / 2$ Mrd $€$. Bis Ende 2013 dürften weitere Mittel, wenn auch im Vergleich zu den Vorjahren aus deutlich geringeren Überschüssen hinzukommen

\section{Deutsche Sozialversicherung im europäischen Haushaltsüberwachungsverfahren}

Mit Blick auf die Einhaltung der europäischen Haushaltsgrenzen für den $\mathrm{Ge}$ samtstaat fielen die Finanzierungssalden der Sozialversicherungen im vergangenen Jahrzehnt kaum ins Gewicht. Vor allem, da faktisch für die öffentlichen Haushalte insgesamt ein Spielraum bis zu $3 \%$ des BIP gesehen wurde, der zudem über viele Jahre hinweg mehr als ausgeschöpft wurde. In struktureller Betrachtung waren die Defizite des Gesamtstaats seit der Jahrtausendwende aber meist größer als die mittelfristig anzustrebenden 0,5\% des BIP. Hier ist die Entwicklung in der Vergangenheit allerdings ein wenig geeigneter Indikator für die Zukunft, weil mit der neuen verfassungsmäßigen Schuldenregel ein neues Regime eingeführt wurde, das die Entscheidungsträger nun $\mathrm{zu}$ einer solideren Finanzpolitik anhalten soll. Außerdem hat das 0,5\%-Ziel des Stabilitäts- und Wachstumspakts mittlerweile durch die Möglichkeit der Sanktionierung und die Übernahme in nationales Recht (Fiskalpakt) an Gewicht gewonnen. Zwar sind die Regeln komplex und beinhalten Ausnahmetatbestände, so dass nicht automatisch mit einer Sanktion zu rechnen ist, wenn die Regelgrenze überschritten wird. Generell sollten die Regeln jedoch nunmehr deutlich härter und bindungsstärker sein. Gerade Deutschland als Stabilitätsanker kommt hier eine besondere Verantwortung zu, eine strikte Regeleinhaltung sicherzustellen.

Die außergewöhnlich hohen Überschüsse des GKV-Systems in den Jahren 2011 und 2012 von $91 / 2$ Mrd $€$ und nochmals 9 Mrd $€$ waren zum kleinsten Teil auf die günstige konjunkturelle Entwicklung zurückzuführen. Entscheidend war vielmehr die (Wieder-)Anhebung des allgemeinen Beitragssatzes von 14,9\% auf $15,5 \%$ zum 1. Januar 2011. Hinzu kamen Ausgabenbegrenzungen wie zusätzliche
Zwangsrabatte von Arzneimittelherstellern, Deckelung der Verwaltungsausgaben der Krankenkassen oder Abschläge bei Mehrleistungen im Krankenhausbereich. Diese im „Gesetz zur nachhaltigen und sozial ausgewogenen Finanzierung der Gesetzlichen Krankenversicherung“ verankerten Regelungen wurden unter dem Eindruck einer sehr ungünstig eingeschätzten Finanzentwicklung in der gesetzlichen Krankenversicherung beschlossen. Da diese Befürchtungen aus heutiger Sicht übertrieben waren, wurde den Krankenkassen im Ergebnis ein beträchtlicher finanzieller Spielraum verschafft, der den Druck zur baldigen Erhebung von $\mathrm{Zu}$ satzbeiträgen verringerte. Das finanzielle Ergebnis dieser Gesetzgebung waren zum größten Teil nicht konjunkturbedingte Überschüsse. So sind diese Salden im Haushaltsüberwachungsverfahren auch in den strukturellen Finanzierungssaldo des Gesamtstaates eingeflossen. Die
Die außergewöhnlich hohen Überschüsse der GKV waren zum kleinsten Teil auf die günstige Konjunktur zurückzuführen.

ungünstigere Lage der übrigen öffentlichen Haushalte wurde damit zum Teil verdeckt. Gleichwohl sind die jüngsten strukturellen Überschüsse des GKVSystems nicht anhaltend. Aufgrund der Ausgabendynamik, die grundsätzlich die der Beitragsbemessungsgrundlage übersteigt, stellen sie letztlich nur ein temporäres Phänomen dar und können nicht ohne weiteres in die Zukunft fortgeschrieben werden.

Nach den hohen Überschüssen der beiden vergangenen Jahre stellt sich nun die Frage, wie rasch sich die Rücklagen

1 Eine Ausnahme stellen die Jahre 2001 bis 2003 dar in denen eine Bruttoverschuldung aller Kassen von 8,3 Mrd $€$ aufgebaut wurde. Dies beruhte allerdings auf zu niedrig festgesetzten Beitragssätzen infolge zu optimistischer Einschätzungen der Entgeltund Beschäftigungsentwicklung. Mit dem GKV-Modernisierungsgesetz wurde den Kassen explizit die Rückführung der aufgelaufenen Verschuldung binnen vier Jahren vorgeschrieben.

2 Konjunkturbereinigungsverfahren beruhen auf Schätzungen, die je nach verwendeter Methode unterschiedlich ausfallen können und deren Ergebnisse überdies im Zeitablauf revisionsanfällig sind. 
in einem normalen, nicht durch weitere diskretionäre Eingriffe der Politik beeinflussten Umfeld voraussichtlich wieder zurückbilden werden. Die vergangene Entwicklung legt die Vermutung nahe, dass dieser Prozess nicht abrupt, sondern eher allmählich in allerdings immer größer werdenden Schritten ablaufen könnte. In den Jahren 1995 bis 2012 sind beispielsweise die Ausgaben der gesetzlichen Krankenversicherung im Jahresdurchschnitt um 2,4\% gestiegen, während die Grundlohnsumme jährlich nur um 1,5\% wuchs. Schreibt man grob vereinfachend das Wachstumsdifferenzial von knapp einem Prozentpunkt in die Zukunft fort, so würden die Überschüsse jedes Jahr zunächst um gut $1 \frac{1}{2} 2 \mathrm{Mrd} €$ gegenüber dem Vorjahr geringer ausfallen. Wenn sich in den Folgejahren die absoluten Ausgaben immer mehr den Einnahmen (bei unverändertem Beitragssatz) angleichen und dann diese schließlich übersteigen, werden die Rücklagen abgeschmolzen und dürften spätestens 2020 vollständig aufgebraucht sein. In diesem Rechenexempel treten aber in der Spitze durchaus strukturelle ${ }^{3}$ Defizite des GKV-Systems in einer Größenordnung von $10 \mathrm{Mrd} €$ auf, mit denen der rechnerische Spielraum mit Blick auf die gesamtstaatliche Haushaltsgrenze von maximal 0,15\% des BIP mehr als ausgenutzt würde. ${ }^{4}$ Die tatsächliche Finanzentwicklung der gesetzlichen Krankenversicherung ist freilich wesentlich komplexer. So verschlechtert sich die Finanzlage derzeit deutlich rascher. Überdies kommt es nicht nur auf die Gesamtentwicklung, sondern die des Gesundheitsfonds und letztlich jeder einzelnen Krankenkasse an, die gegebenenfalls Zusatzbeiträge erheben müssen.

Wenn durch das vorübergehende Abschmelzen der Rücklagen das mittelfristige Haushaltsziel verfehlt zu werden droht, ist von einer vorausschauenden Finanzpolitik zu erwarten, dass auf den übrigen Haushaltsebenen rechtzeitig vorgesorgt wird, beispielsweise indem der Bund sein strukturelles Defizit zumindest vorübergehend unter $0,35 \%$ des BIP hält.

\section{Sanktionen für Deutschland zu befürchten?}

Tatsächlich erscheint eine Maßregelung Deutschlands Im Rahmen des europäischen Haushaltsüberwachungsverfahrens nicht aber sehr wahrscheinlich.
Da das Mittelfristziel eines strukturellen Defizits von $0,5 \%$ des BIP bereits 2012 mit einem Überschuss von $0,4 \%$ erreicht wurde und ausweislich des im Frühjahr 2013 aktualisierten Stabilitätsprogramms bis 2017 weiter mit einem ab 2014 konstanten strukturellen Überschuss von $1 \frac{1}{2} \%$ des BIP mit einem Sicherheitsabstand eingehalten werden soll, geht die Bundesregierung offensichtlich davon aus, dass die absehbaren Defizite in der gesetzlichen Kranken- (und Renten-) Versicherung durch noch größere Überschüsse bei Bund, Ländern und Gemeinden überkompensiert werden.

\section{Die Kosten einer potenziellen Maßregelung durch die EU sind weniger finanzieller als politischer Natur.}

Das europäische Überwachungsverfahren sieht selbst dann, wenn die finanzpolitischen Entscheidungsträger im Deutschland von ihrem Mittelfristkurs wieder abkommen sollten, noch mehrere Zwischenschritte bis zur Verhängung von Sanktionen vor. Bis eine finanziell spürbare Sanktionierung greift, muss ein komplexer Entscheidungsprozess durchlaufen werden, der vor allem zum Ziel hat, das betreffende Mitgliedsland zur Rückkehr zu einer soliden Finanzpolitik zu bewegen. Die Strafandrohung soll also selbst im Defizitverfahren noch präventiv wirken. Die Kosten einer potenziellen Maßregelung durch die Gemeinschaft sind daher auch weniger finanzieller als vielmehr politischer Natur - soweit mit einer als unsolide beurteilten Finanzpolitik im jeweiligen Fall ein Reputationsverlust verbunden ist.

Im „präventiven Arm“ des Stabilitätsund Wachstumspakts muss die Europäische Kommission zunächst in ihrer ex post Analyse eine „signifikante Abweichung" vom Anpassungspfad zum Mittelfristziel feststellen. Dies ist dann der Fall, wenn das mittelfristige Haushaltsziel und die angemessene Verringerung des strukturellen Defizits in einem Jahr um $0,5 \%$ oder um mindestens $0,25 \%$ des BIP in zwei aufeinander folgenden Jahren verfehlt wird. Außerdem müssen dabei die Ausgaben schneller gestiegen sein als eine angemessene Referenzrate und diese Abweichung muss einen Effekt von mindestens $0,5 \%$ des BIP in einem
Jahr oder um mindestens 0,25\% des BIP in zwei aufeinander folgenden Jahren haben. Sofern eines dieser zwei Kriterien klar verfehlt wird, kann die Kommission in einer Gesamtwürdigung eine signifikante Abweichung feststellen. Sie richtet daraufhin eine Verwarnung an den Mitgliedstaat. Es folgt dann innerhalb eines Monats eine entsprechende Empfehlung des Rates (auf Empfehlung der Kommission), Gegenmaßnahmen (innerhalb von normalerweise 5 Monaten) zu ergreifen. Sodann prüft die Kommission, ob effektive Maßnahmen ergriffen wurden, um zum Mittelfristziel zurück zu gelangen. Wird dies von der Kommission verneint, kann der Ministerrat dies mit qualifizierter Mehrheit bestätigen. Kommt diese Entscheidung im Ministerrat nicht zustande, legt die Kommission eine neue Empfehlung vor, die der Rat mit einfacher Mehrheit ablehnen kann („umgekehrte einfache Mehrheit"). Nimmt der Rat die Entscheidung an, wird der Weg zu Sanktionen grundsätzlich frei. Die Kommission empfiehlt eine verzinslichen Einlage ${ }^{5}$ in Höhe von $0,2 \%$ des BIP (also aus derzeitiger Sicht rund 51/2 Mrd $€$ im Falle Deutschlands). Diese Sanktionsempfehlung ist angenommen, wenn sie nicht vom Rat mit qualifizierter Mehrheit abgelehnt wird („umgekehrte qualifizierte Mehrheit“).

Zusätzliche Ermessensspielräume eröffnen sich für die Kommission schließlich durch zwei Ausnahmetatbestände. So

3 Die steigenden Defizite des GKV-Systems sind spiegelbildlich zum Rücklagenaufbau überwiegend als strukturell zu werten, da dieser fundamentale Prozess unabhängig von der konkreten Konjunkturentwicklung abläuft bzw. konjunkturell nur überlagert wird. Eine Klassifizierung als temporäre und somit nicht strukturelle Defizite wäre insofern inkonsistent, als auch die Überschüsse im Vorfeld nicht als temporär eingestuft worden waren.

4 In Bezug auf das 0,5 \%-Kriterium des Fiskalpakts ist zu beachten, dass hier BIP-Quoten ausschlaggebend sind. Bei steigendem nominalen BIP steigt der 0,15\% entsprechende Euro-Betrag für das maximal zulässige strukturelle Defizit außerhalb des Bundeshaushalts.

5 Der Zinssatz soll gem. Regulation (EU) 1173/2011, Artikel 4, das Kreditrisiko der Europäischen Kommission und damit letztlich des EU-Haushalts widerspiegeln. Beispielsweise konnte sich die Rettungsfazilität EFSM (Europäischer Finanzstabilitätsmechanismus) zuletzt (Ende 2012) mit $2,5 \%$ finanzieren. Kosten für die Steuerzahler eines Mitgliedslandes entstehen daher in dem Fall, in dem die nationalen Finanzierungskosten jene der Europäischen Kommission übersteigen. Im umgekehrten Fall entstehen Erträge. 
kann auf die Empfehlung einer Sanktion verzichtet werden, wenn ein außergewöhnlicher Effekt außerhalb der Kontrolle des Mitgliedstaates oder ein schwerer Wirtschaftsabschwung in der EU insgesamt festgestellt wird. Diese Ausnahmefälle dürften für strukturelle Defizite infolge des Rücklagenabbaus deutscher Sozialversicherungen aber kaum einschlägig sein. Zudem kann aber auch die Kommission aufgrund eines begründeten Antrags des betreffenden Mitgliedstaates dem Rat eine Verringerung oder Aufhebung der verzinslichen Einlage empfehlen.

Endgültige Strafzahlungen kommen erst im „korrektiven Arm“ des Stabilitäts- und Wachstumspakts in Betracht. Dazu muss zunächst ein Verfahren bei einem übermäßigen Defizit eingeleitet worden sein, was wiederum eine geplante oder gemeldete (unbereinigte) Defizitquote von über $3 \%$ oder eine unzureichende Rückführung der Schuldenquote von über $60 \%$ voraussetzt. In einem mehrstufigen Abstimmungsprozess zwischen der Europäischen Kommission und dem Europäischen Rat kann zunächst eine unverzinsliche Einlage von 0,2\% des BIP verlangt werden. Dies wäre bei den aktuell niedrigen Refinanzierungskosten für Deutschland mit einem Zinsverlust in Höhe von rund 55 Mio $€$ verbunden. Die Abschreckungswirkung wäre auch hier in erster Linie politischer Natur. Bei anhaltender Nichtbefolgung von Empfehlungen (Nicht-Ergreifen von wirksamen Maßnahmen zur Korrektur des übermäßigen Defizits) kann eine Geldbuße in Höhe von 0,2 \% des BIP verhängt werden. Im weiteren Verlauf (Nichtbefolgung der Inverzugsetzung) kann eine mögliche jährliche Geldbuße höher ausfallen, aber nicht $0,5 \%$ des BIP überschreiten.

Die innerstaatliche Verteilung von Sanktionszahlungen ist im Grundgesetz (Art. 109 Abs. 5) und im Sanktionszahlungs-Aufteilungsgesetz geregelt. Danach müsste der Bund $65 \%$ und die Länder $35 \%$ tragen. Der Länderanteil würde wiederum zu 35 \% nach der Einwohnerzahl und zu $65 \%$ nach dem Beitrag der einzelnen Länder zum Finanzierungsdefizit der Ländergesamtheit verteilt. Für die Sozialversicherungen ist keine Beteiligung vorgesehen. Im FiskalpaktUmsetzungsgesetz ist als weitere Besonderheit geregelt, dass der Bund bis 2019 Sanktionen im Rahmen des „präventiven Arms“ des Stabilitäts- und Wachs- tumspakts alleine trägt, da die Länder laut nationaler Schuldenbremse erst $\mathrm{ab}$ 2020 einen strukturell ausgeglichenen Haushalt aufweisen müssen. Wie oben ausgeführt, droht hier aber lediglich eine verzinsliche Einlage, die nur dann mit Kosten verbunden wäre, wenn die Refinanzierungskosten der bestraften Einleger höher wären als die dortige Verzinsung.

\section{Perspektiven für die Finanzbeziehung zwischen dem Bundeshaushalt und den Sozialversicherungen}

Die Verantwortung für die Einhaltung der auf den Gesamtstaat zielenden europäischen Fiskalregeln liegt in erster Linie beim Bund. Hier gilt es nach Erreichen einer ausgeglichenen strukturellen Haushaltsposition die Entwicklungen auf allem Ebenen kritisch zu verfolgen und gegebenenfalls gesetzgeberisch gegenzusteuern. Das grundsätzliche Verschuldungsverbot der Sozialversicherungen erleichtert diese Aufgabe. Steigen die Ausgaben dauerhaft schneller als die beitragspflichtigen Einkommen, können Beitragserhöhungen nur dann durch Bundeszuschüsse vermieden werden, wenn dort eine nachhaltige Gegenfinanzierung durch Einsparungen an anderer Stelle oder Steuererhöhungen sichergestellt ist. Grundsätzlich ist aber darauf hinzuweisen, dass idealerweise Steuerzuschüsse nur zur Finanzierung ,versicherungsfremder Leistungen“ verwendet werden sollten, um $\mathrm{zu}$ verhindern, dass die Gemeinschaft der Beitragszahler gesamtgesellschaftliche Aufgaben finanzieren muss. Diese Betrachtung gilt allerdings vor allem in den Sozialversicherungszweigen mit einem hohen Grad an Beitragsäquivalenz, insbesondere also der gesetzlichen Rentenversicherung. In der gesetzlichen Krankenversicherung steht der (begrenzt) einkommensabhängige Beitrag dagegen in keinem proportionalen Verhältnis zum Umfang des damit erworbenen Versicherungsschutzes. Gleichwohl erscheint eine Begründungspflicht des Gesetzgebers im Sinne der „versicherungspflichtigen Leistungen“ angebracht, um die Versichertengemeinschaft vor willkürlichen Be- und Entlastungen zu schützen.
Wenngleich das Risiko, im Rahmen der Haushaltsüberwachungsverfahren tatsächlich in Haftung genommen zu werden, für die Sozialversicherungen im Allgemeinen und die gesetzliche Krankenversicherung im Besonderen sehr überschaubar scheint, dürften die enger gewordenen finanziellen Spielräume im Bundeshaushalt doch dazu führen, dass die Finanzbeziehungen zwischen dem Bund und dem System der gesetzlichen Krankenversicherung auch künftig immer wieder auf den Prüfstand gestellt werden. Dabei wurde schon bislang der Bundeszuschuss an die Krankenkassen bzw. seit 2009 an den Gesundheitsfonds sehr häufig finanz- und wirtschaftspolitischen Notwendigkeiten angepasst. Dauerhafte, geeignete Regeln mit verlässlicher Bindungswirkung wurden hier bislang nicht etabliert.

Erstmals wurde ein Bundeszuschuss (I) mit dem GKV-Modernisierungsgesetz eingeführt. Durch ihn sollten versicherungsfremde Leistungen aus Steuer- und nicht mehr aus Beitragsmitteln finanziert werden. Das Volumen wurde seinerzeit pauschal auf 4,2 Mrd $€$ taxiert. In Zwischenschritten von 1 Mrd $€$ im Jahr 2004 und 2,5 Mrd $€$ im Folgejahr sollte ab 2006 der Endwert erreicht werden. Eine Dynamisierung war nicht vorgesehen.

\section{Nachvollziehbare Regeln mit verlässlicher Bindungswirkung wurden beim Bundeszuschuss an die GKV bislang nicht etabliert.}

Es fehlte auch eine genaue Aufschlüsselung darüber, was der Gesetzgeber konkret unter „versicherungsfremden Leistungen“ verstand. Für das Jahr 2007 wurde der Zuschuss zur Entlastung des Bundeshaushalts wieder auf 2,5 Mrd $€$ zurückgenommen und sollte danach ganz entfallen. Die nächste Kehrtwende folgte mit dem GKV-Wettbewerbsstärkungsgesetz, durch das die Bundesmittel auf einen Zielwert von 14 Mrd $€$ angehoben wurden (Bundeszuschuss II). Dieser neue Endwert sollte in Steigerungsschritten von 1,5 $\mathrm{Mrd} €$ pro Jahr allmählich erreicht werden. Im Rahmen der Konjunkturprogramme wurde der allgemeine Beitragssatz zur Jahresmitte 2009 von 15,5 \% auf 14,9\% 
gesenkt. Die dadurch verursachten Einnahmenausfälle von gut $6 \mathrm{Mrd} €$ pro Jahr wurden wiederum durch ein Vorziehen der Erhöhungsstufen mit Steuermitteln ausgeglichen. Hinzu kamen einmalige Sonderzuschüsse von knapp $4 \mathrm{Mrd} €$ im Jahr 2010 und 2 Mrd $€$ im Folgejahr. Dadurch erreichte der Bundeszuschuss im Jahr 2010 sein bisheriges Maximum von 15,7 Mrd €. Bis 2012 fiel er wieder auf seinen langfristig anvisierten stabilen Wert von 14 Mrd €. Doch bereits 2013 wurde wiederum zur Entlastung des Bundes eine Kürzung um 2,5 Mrd € vorgenommen, die 2014 noch um 1 Mrd $€$ ausgeweitet werden soll. Im Jahr 2015 soll der Bundeszuschuss wieder auf 14 Mrd $€$ angehoben werden. Die bisherige Geschichte des Bundeszuschusses belegt eindrucksvoll seine Unstetigkeit und damit letztlich wohl auch seine politische Unzuverlässigkeit.

Aus Sicht des Bundes bedeutet die vorübergehende Kürzung der Zuschüsse an den Gesundheitsfonds (und auch an die gesetzliche Rentenversicherung und - per saldo - an die Bundesagentur für Arbeit) eine „strukturelle“6 Verbesserung seiner Haushaltsposition. Nachhaltig ist diese Haushaltsentlastung jedoch nicht, wenn die Rückkehr zum anvisierten Zuschussniveau ernst genommen wird.

Überdies sollte der Bund ab 2015 Überweisungen an den Gesundheitsfonds zur Finanzierung des sozialen Ausgleichs leisten. Eine Überforderung der GKV-Mitglieder wird immer dann unterstellt, wenn der - per Schätzung ermittelte - durchschnittliche Zusatzbeitrag $2 \%$ der individuellen beitragspflichtigen Einnahmen übersteigt. Die Differenz wird dem Mitglied durch entsprechende Minderung seines einkommensabhängigen Beitrags erstattet. Die dadurch verursachten Einnahmenausfälle beim Gesundheitsfonds sollten bis 2014 aus den Rücklagen des Fonds gespeist werden. In den Jahren danach war der Bund zur Übernahme dieser Kosten verpflichtet. Bis einschließlich 2013 wurde jedoch noch kein durchschnittlicher Zusatzbeitrag festgelegt, weil die vom Gesundheitsfonds den Kassen überwiesenen Mittel bislang ausreichten, um deren Ausgaben vollständig zu decken. Die große Koalition beabsichtigt nun jedoch, den Zusatzbeitrag einkommensproportional zu gestalten. Die Bundesmittel für den sozialen Ausgleich würden damit hinfällig.

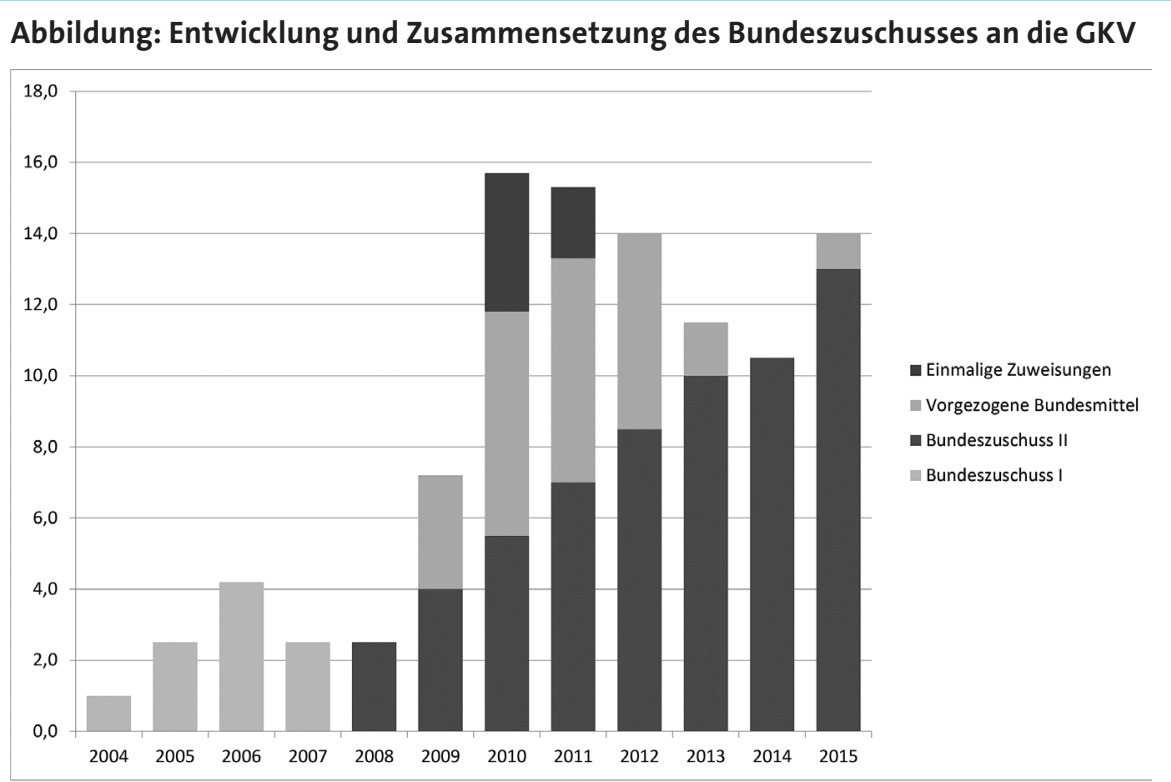

Quelle: Deutsche Bundesbank

Die bisherigen Erfahrungen mit den Zuschüssen des Bundes an die gesetzliche Krankenversicherung lassen Zweifel an deren verlässlicher Planbarkeit aufkommen. Vor dem Hintergrund der nationalen und europäischen Haushaltsregeln ist dem Bund der bislang übliche einfache Weg über die zusätzliche Staatsverschuldung verbaut. Sollten sich noch Konsolidierungsnotwendigkeiten ergeben, dürften erneut kurzfristig realisierbare Einsparpotenziale gesucht werden. Hier ist der Spielraum eher eng, da vertraglich oder gesetzlich festgelegte Zahlungsversprechen nicht ohne Weiteres zur Disposition gestellt werden können. Ein beliebter Kandidat sind die Investitionsausgaben und - wie die Erfahrung lehrt - Verschiebebahnhöfe zu den Sozialversicherungen.

Die gesetzliche Krankenversicherung wird von zwei Seiten aus gleicher Ursache unter Druck geraten. Einerseits steigt bei ihr selbst der Ausgabendruck infolge der demographischen Veränderungen wenngleich die Zusammenhänge hier wesentlich komplexer und folglich weniger eindeutig zu prognostizieren sind als etwa im Bereich der gesetzlichen Rentenversicherung. Hinzu kommt, dass die Einnahmenbasis geschwächt wird, wenn die Zahl der Beitragszahler ab etwa Mitte des laufenden Jahrzehnts kontinuierlich abnehmen wird. Andererseits wird auch der Bundeshaushalt insbesondere durch eine schrumpfende Steuerbasis unter der Bevölkerungsalterung verstärkt unter Druck geraten.
Insgesamt sind die finanziellen Aussichten für die gesetzliche Krankenversicherung mittelfristig nicht günstig. Gerade dann, wenn ihre Rücklagen aufgebraucht sein werden und der eigene Ausgabendruck merklich steigen wird, besteht kaum noch Hoffnung auf höhere oder auch nur stabile Zuschüsse aus dem Bundeshaushalt. Folglich werden Lösungen innerhalb des Systems der Krankenversicherung gefunden werden müssen. Anders ausgedrückt: Die Verteilungskonflikte zwischen und innerhalb der am Gesundheitssystem beteiligten Gruppen (Mitglieder, Arbeitgeber und Leistungserbringer) könnten wieder härter ausfallen. Indirekt ist dies auch eine Folge des Fiskalpakts. Die durch Haushaltsregeln angestrebte solide Finanzpolitik ist allerdings insbesondere angesichts der demographischen Perspektiven unverzichtbar. Eine Flucht in die staatliche Verschuldung ist keine tragfähige Option.

6 Maßgeblich für die Schuldenbremse des Grundgesetzes ist die um konjunkturelle Effekte und finanzielle Transaktionen (z.B. Beteiligungsveräußerungen, Darlehensvergaben) bereinigte Nettokreditaufnahme des Bundes. 